UCRL-JC-107391

PREPRINT

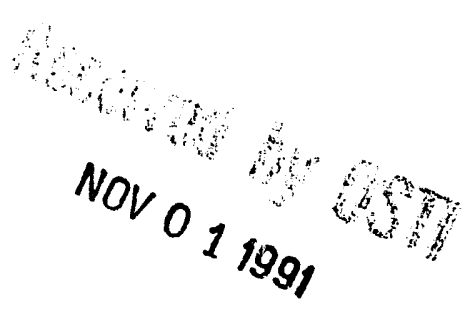

\begin{abstract}
Remote Experimental Site: A Command and Analysis
Center for "Big Physics" Experimentation
\end{abstract}

Thomas A. Casper and William J. Lennon
14th IEEE Symposium on Fusion Engineering
San Diego, CA
September 30 - October 3, 1991

September 1991
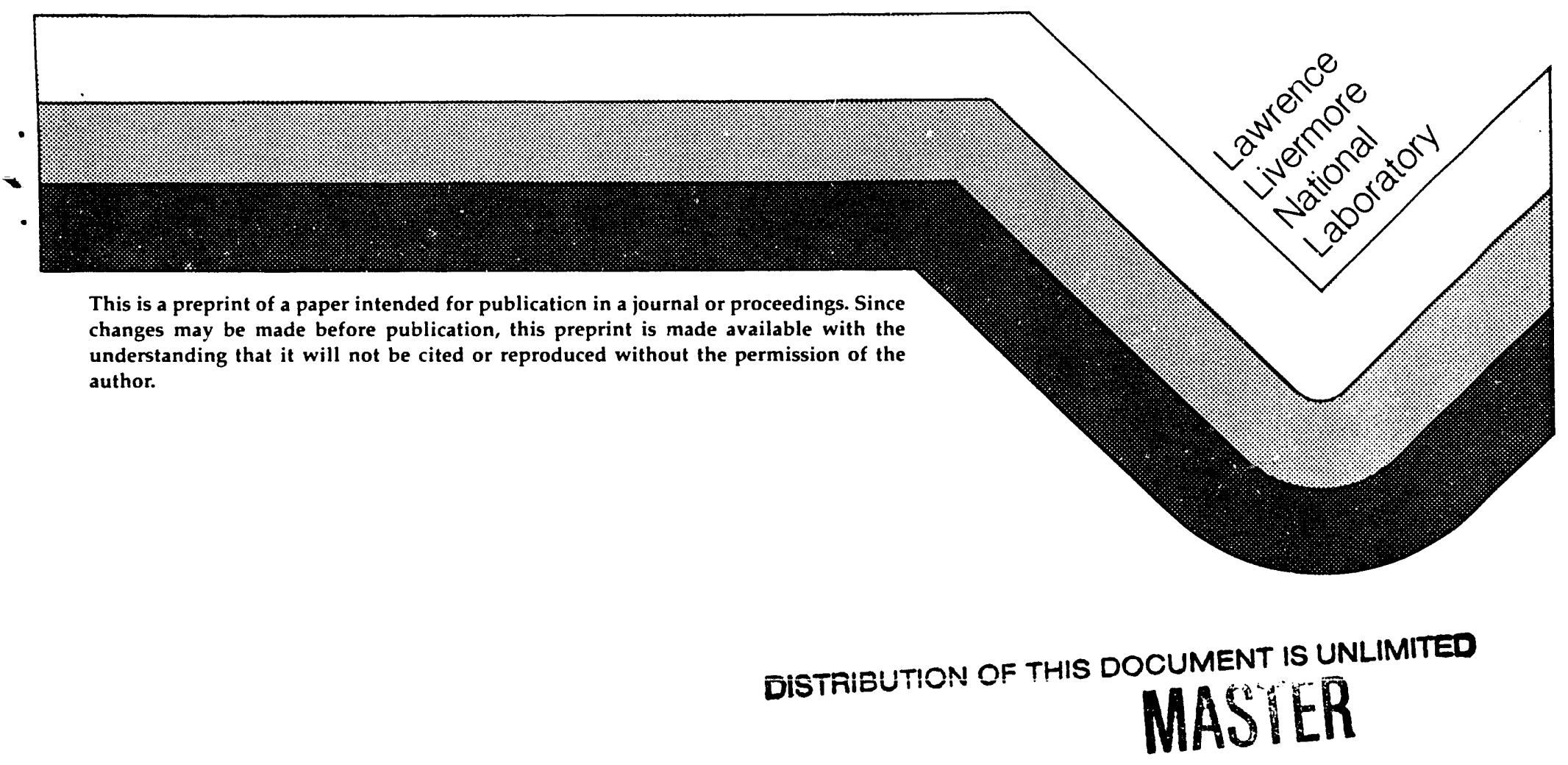


\section{DISCLAIMER}

This document was prepared as an account of work sponsored by an agency of the United States Government. Neither the United States Government nor the University of California nor any oi their employees, makes any warranty. express or implied, or assumes any legal liability or responsibility for the accuracy; completeness, or usefulness of any information, apparatus, product, or process disclosed, or represents that its use would not infringe privately owned rights. Reference herein to any specific commercial products, process, or service by trade name, trademark, manufacturar, or otherwise, doces not necessarily constitute or imply its endorsenent, recommendation. or favoring by the United States Government or the University of California. The views and opinions of authors exprexsed herein do not necessarily state or reflect those of the United States Government or the University of California, and shall not be used for advertising or product endorsement purposes. 


\title{
REMOTE EXPERIMENTAL SITE: A COMMAND AND ANALYSIS CENTER FOR "BIG PHYSICS" EXPERIMENTATION
}

\author{
Thomas A. Casper and William J. Lennon \\ Lawrence Livermore National Laboratory \\ University of California \\ P.O. Box 5511, Livermore, California, 94550, USA
}

\begin{abstract}
The next generation of tokamaks, ITER or BPX, will be characterized by an even greater emphasis on joint operation and experimentation. With anticipation of an increased number and diversity of collaborations, we are preparing for such shared facilities by developing a systematic approach to remote, joint physics operation involving experimental teams at several locations. The local area network of computers used for control and data acquisition on present and future experiments can be extended over a wide area network to provide a mechanism for remote operation of subsystems required for physics experiments. The technology required for high bandwidth ( $\geq 45 \mathrm{Mbps})$ connections between multiple sites either exists or will be available over the next few years. With the rapid development of high performance workstations, network interfaces, distributed computing, and video conferencing, we can proceed with the development of a system of control and analysis sites to provide for consistent, efficient, and continuing collaborations. Early establishment of such sites could also enhance existing joint design and development efforts.

\section{INTRODUCTION}

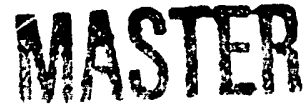

As fusion research approaches reactor conditions, the trend is towards larger and fewer experimental facilities, leaving many major institutions without a local experiment. The problem of integrating those experimental teams into the ongoing fusion program is an important one, since the expertise and experience is quite valuable. To sustain a continued involvement in experiments, scientists are required to travel frequently, or to move their families to the new large facilities. This problem is common to other fields of research, such as high energy physics, materials science, and astronomy.

The next phase in the fusion experimental program calls for the dramatic increase in the size and cost of both inertial and magnetic confinement experiments. Treating these large physics experiments as "(inter)national
\end{abstract}


facilities" to be operated and utilized by several groups at different institutions maintains a healthy diversity of scientific expertise. National laboratory involvement prorides the added dimension of technological and engineering expertise extracted from diverse programs. University involvement insures a continuing supply of new talent and ideas. Local industrial participation insures the transfer of technology and the development of future comrnercial capabilities.

We have initiated a program to develop a "Remote Experimental Site (RES)" concept to link geographically separated institutions over high speed, wide area networks based on fibre optic transmission which are rapidly being developed and implemented. Use of such remote sites could dramatically alter the approach to collaborations on expensive, one-of-a-kind, high-technology devices. Rather than sending teams of experts for extended operational periods, institutions could be connected integrally with the facility, in remote sites that in every way resemble the local control room at the machine site. Distributed operation and data analysis would occur in real time from the local site and the set of several remote sites.

The Remote Experimental Site (RES) is a concept for the distributed operation of a facility from a remote location, indicated schematically in Figure 1. It uses the technology of high-speed digital data transfer over a wide area network (WAN) to provide near real-time transfer of experimental data. Fast response is required, for example, for control of a subsystem or diagnostic, and for analysis and data display that may affect experimental operations. In addition, to foster closer personal interaction, provisions are incorporated for voice and video conferencing between the experimental facility and the RES. This is to provide the "look and feel" of local operations to the remote group. It provides the essential information for operations, diagnostics displays, and fault conditions and allows for physics discussions relevant to the experiment.

\section{APPLICATIONS TO MAGNETIC FUSION RESEARCH}

The operation of next-generation tokamaks, such as the Burning Plasma Experiment (BPX) and the International Thermonuclear Experimental Reactor (ITER), will produce fluxes of fusion reaction products sufficient to activate the surrounding structures. A direct consequence of this successful operation will require the use of remote procedures and robotics and will allow only brief and limited access to the vessel area, even for the staff located at the experiment. As sketched in Figure 1, the control room, data acquisition facilities, and work areas will be remotely located from the experiment and isolated by large biological barriers. It is anticipated that connections to the experiment will be over fiberoptic highways for visual display and computer controls and diagnostics connections. It is only a minor technological extrapolation to extend these connections to other, perhaps several, institutions that are geographically remote from the location of the experiment itself.

For early testing of this RES concept, we are implementing such an interactive control link to the DIID tokamak at General Atomics as an extension of experimental operations associated with an existing GA/LLNL collaboration. Utilization of the RES prototype for these collaborative experiments requires 


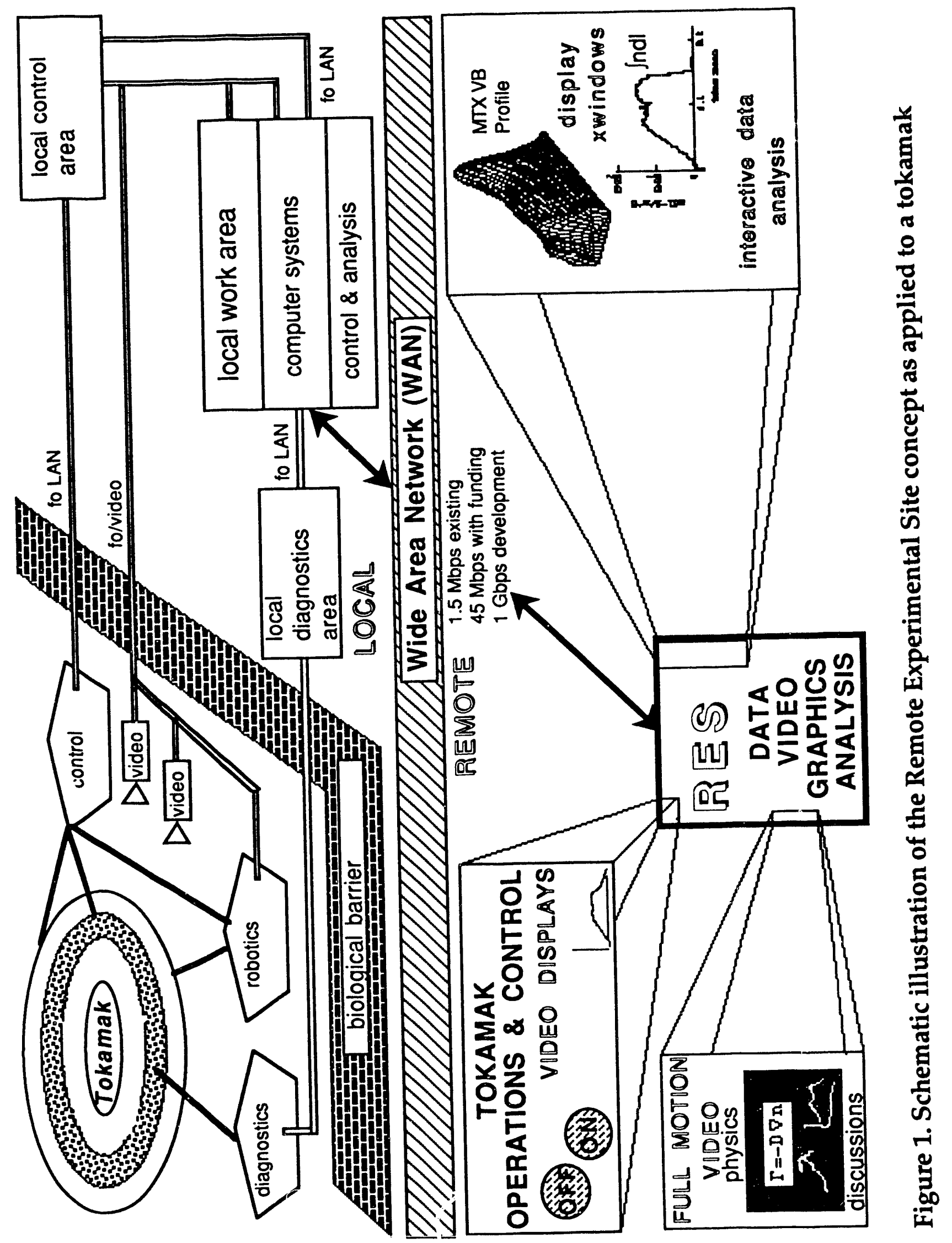


commitment by the local computer group to provide system modifications such as network interfaces into their database, possible additions to their display techniques, and accommodations to include the remotely interacting group at LLNL. These early proof of principle experiments will identify critical performance constraints while addressing some of the sociological issues inherent with such remote collaborations. The initial configuration of our developmental RES is shown in Figure 2. Most components for this limited RES are presently available or soon to be delivered. Utilizing this configuration, we can explore issues related to performance and display and can implement network bandwidth enhancements as they become available. Full implementation of the first fusion RES at LLNL requires additional funding and development for extensions beyond the design and testing phase described here. Some effort to increase network throughput may be necessary at that time to provide useful, interactive response.

Industrial development of packetized video and multimedia workstations (interfaces for integrated video and data) is at present rapidly accelerating with commercial products expected to be available in the 1993-1994 time frame. We wish to stimulate these developments and contribute to the establishment of evolving standards in this area. For that reason, we invite industrial participation in developing the ideas for implementing the concept. Achievement of full integration of data and packetized, full motion video requires use of advanced network applications such as fiber distributed data interface (FDDI) for a 100Mbps LAN and synchronous optical network (SONET) for the high speed WAN. Implementation of proposed upgrades to the Energy Sciences network (ESnet) performance to accommodate the video and data integration would be most beneficial to this development and critical to a multiple RES environment. It can provide the future underlying WAN for the transparent, parallel connection of several sites.

Design and development of the complete RES concept including high speed WAN and video-conferencing is certainly applicable to both the BPX and ITER experiments. The ITER/BPX application would best be accomplished through collaboration in the early design phase for operations, control, and data acquisition systems. Development for BPX could serve as a prototype for the ITER experiment. Upgrades of the RES to take advantage of commercial computer and network advances and to accommodate the ITER international scale could be done during the development phase of the ITER project. Early implementation would directly benefit the engineering design effort itself.

The fundamental design of our existing data acquisition and control system used by the MTX experiment at LLNL, Figure 3, is based on the LAN concept with computers synchronized via a process-to-process message transfer technique, our own interprocess communications system (IPCS) ${ }^{1}$. This LAN includes our office automation system (mostly AppleTalk and VAX connections), link to the National Energy Research Supercomputing Center (NERSC), tokamak data acquisition on UNIX workstations connected with a VMS VAX-cluster, and UNIX workstations for control of (newer) tokamak systems. The system design allows for remote operation of the MTX tokamak itself and of various diagnostic and tokamak subsystems. Network access to the data and limited display of operations information are an integral part of our 


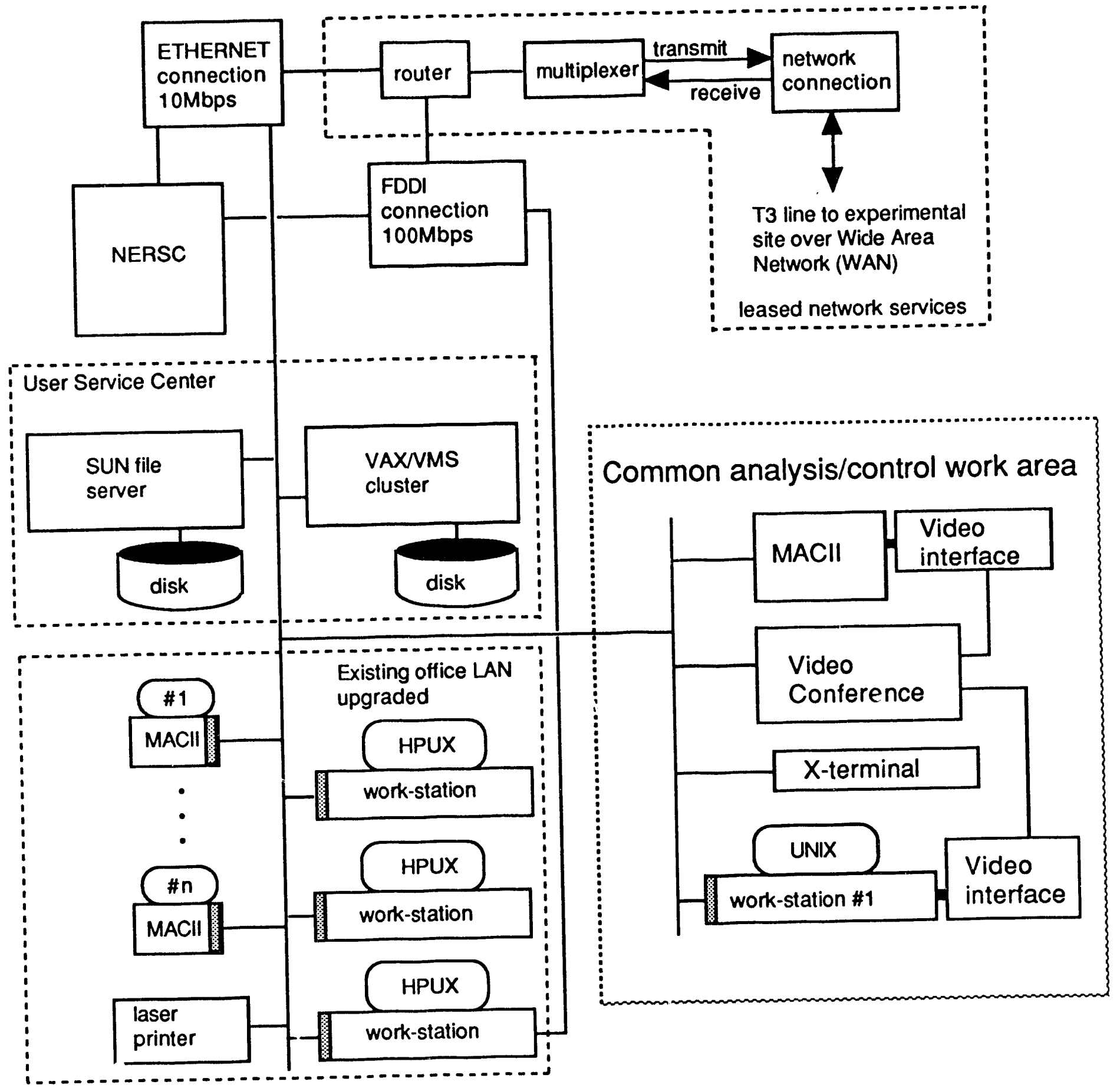

Figure 2. Initial RES configuration implemented at LLNL 


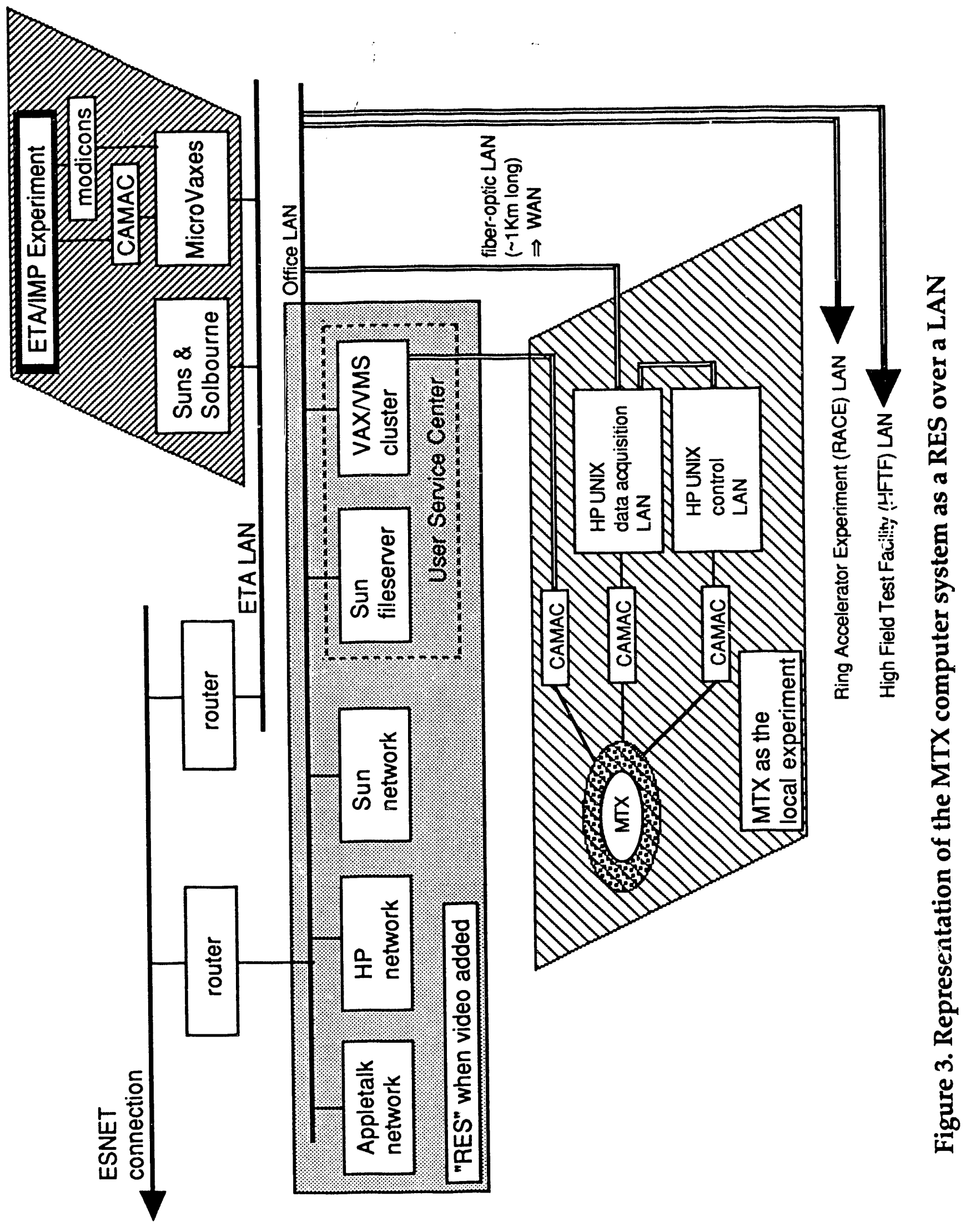


system. Extensions for additional display of operations information and safety interlocks could easily be added. This system design and network configuration is readily amenable to RES operation. The liberal use of workstations for acquisition and control is very attractive for maintaining system performance, expansibility, and accommodation of collaborators. Tokamak subsystems can be designed, developed, and operated at a remote site before being delivered to the experimental facility. Upon arrival, full incorporation into the operations and utilization of the additional computational power can be achieved in a brief period of time, as short as a few minutes, provided one adheres to certain industry standards.

\section{DEVELOPMENT ISSUES}

An RES would comprise a collection of computer workstations connected in a local area network (LAN), initially an ethernet operating at $10 \mathrm{Mbits} / \mathrm{s}$, which is in turn connected to the experimental facility by a high speed WAN. Experimental computer networks, e. g. the National Research and Education Network (NREN) in the US, are being designed for speeds in excess of $1 \mathrm{Gbit} / \mathrm{s}$. Internal development at LLNL is actively pursuing and has already established credibility in the area of high speed, Gbit/s, networks ${ }^{2}$. There is, however, existing commercial bandwidth capability, $45 \mathrm{Mbits} / \mathrm{s}$, sufficient to accommodate a moderately large volume of data for real time operations plus a limited number of channels of compressed full-motion video.

Workstations would be used for local analysis and display of experimental data in near real time, limited only by the available data transfer rates, and would be fully integrated into the operations and its database. Applications of network distributed computing, presently an active area of research at LLNL, would insure that this distributed computational power is available to and directed by the experimental facility as needed. It is the distributed computational aspects which provides the cost effectiveness of the RES approach to future experiments. This requires successful development of WAN-based distributed computing and software tools and transaction processing of large datasets among possibly different databases. Without the distributed computing aspect, the RES would be little more than a collection of video enhanced remote terminals.

The video link consists of both group conferencing capabilities and the ability for more personal interaction between collaborators or between a remote collaborator and the experimental operations group. Initially, existing commercial video-conferencing systems will provide the necessary medium to suppcrt the general group interactions and discussions. These capabilities provide the ability to view personnel and manual displays (such as blackboards and overhead projectors) as needed for discussion of the operations plans or developing physics. Additional visual and audio communication in support of real time collaborative experiments will ultimately be implemented at the workstation level. This will be achieved through a system of integrated voice, video, and data communications over the WAN. Through the capability to display video, this multimedia workstation approach will provide for closer personal interaction plus the ability to readily view displays monitoring the experimental data, operations, and safety information. 
With a properly designed database and computer network at the experimental facility, sufficient bandwidth over the communication link, and recent computer advances in workstation performance and $\mathrm{X}$-windows interfaces, most display functions could be handled directly with computer workstations of moderate price and performance. Typical performance/cost in the range of $\sim 10 \mathrm{Mips} / \$ 7,000$ to $\sim 75 \mathrm{Mips} / \$ 25,000$ are presently available. Special purpose visualization systems can be added at higher costs. The simplest RES would involve only a single networked workstation, whereas a full-scale RES might include a dedicated operations room connected to a network of computerized offices. It is conceivable that there would be a full-scale RES at many of the major fusion research centers. Much of the existing computer equipment could be upgraded and used with enhancements in the areas of high speed LAN interfaces and digital data and video communications.

In summary, the RES concept has the potential to fundamentally change the manner in which collaborations in fusion and other areas of research are carried out. Collaborations would proceed more cost effectively, and the energies and ideas of different groups would be more easily integrated to significantly enhance the productivity of the research on these large facilities.

\section{REFERENCES}

1. D.N. Butner, T.A. Casper, M.D. Brown, M. Drlik, W.H. Meyer, and J.M.Moller, Rev. Sci. Instrum., 61, 3277 (1990).

2. P. Rupert, "What's Driving Gigabit/second Channels?," in High Speed Fiber Networks \& Channels, K.Annamalai, Editor, Vol 1577, SPIE 1991, ISBN \# 0819407089 

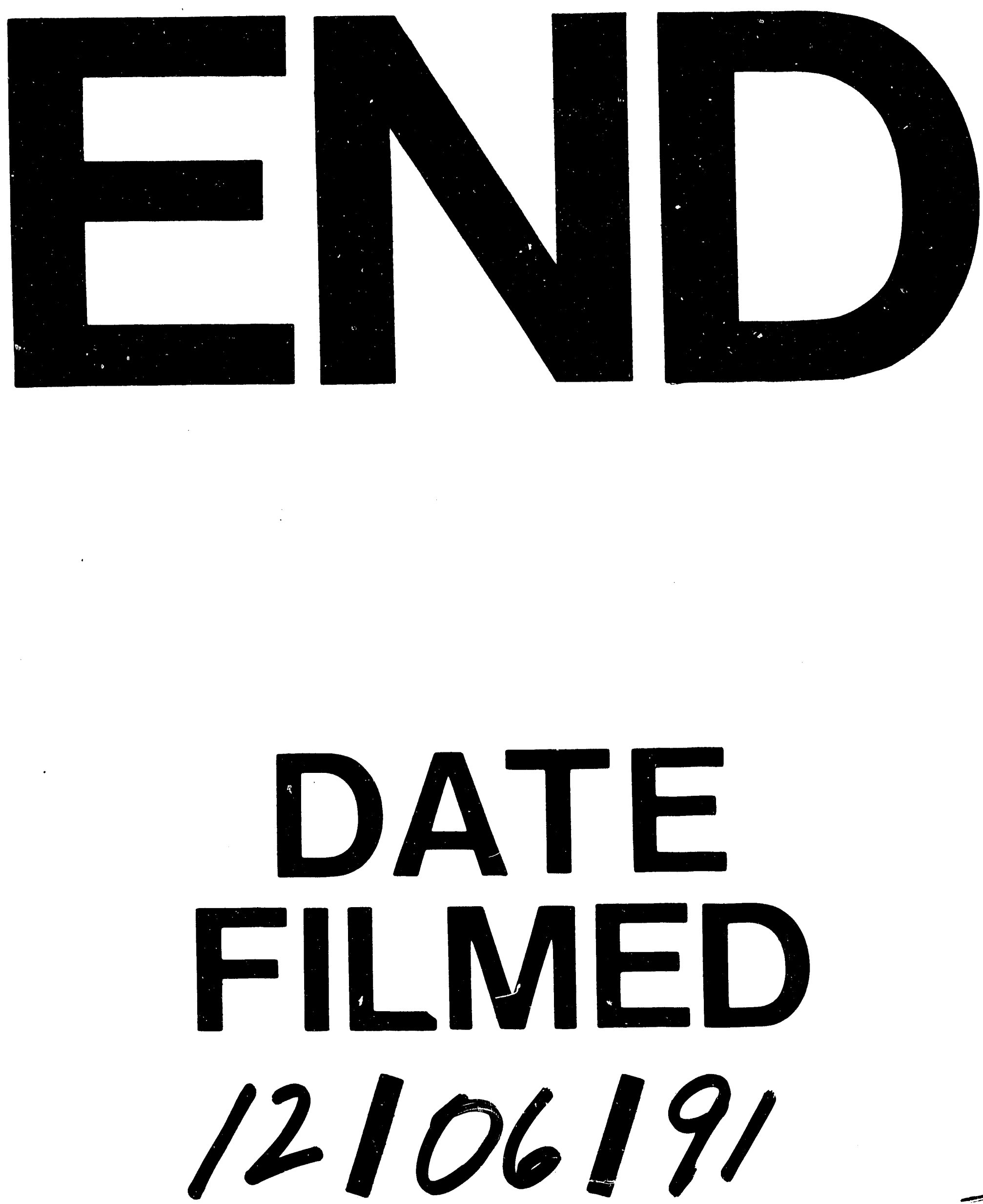

I 


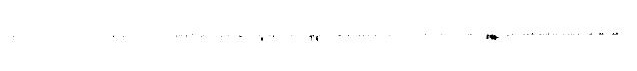

